\title{
Naming Persons in News Videos with Label Propagation
}

\author{
Phi The Pham, Tinne Tuytelaars, Member, IEEE and Marie-Francine Moens, Member, IEEE
}

\begin{abstract}
Labeling persons appearing in video frames with names detected in a corresponding video transcript helps improving video content annotation and search tasks. We implement a face naming method that learns from labeled and unlabeled examples using iterative label propagation in a graph of connected faces or name-face pairs. By incorporating the unlabeled data points during the learning process, this method can work with few labeled data points. Moreover, we present variations of this model that better cope with a large number of data by reducing the time and space complexity. On BBC News videos, the label propagation algorithm yields better results than a Support Vector Machine classifier and a nearest neighbor classifier trained on the same labeled data. Furthermore, we show that when anchor detection precedes the label propagation process, it helps boosting the face naming performance. Reusing labeled examples from different broadcasts, we manage to name $70 \%$ of the faces with a precision of $85 \%$.
\end{abstract}

Index Terms-Cross-media mining, label propagation, video annotation, face identification.

\section{INTRODUCTION}

Massive digitisation efforts combined with the advent of media sharing websites and the explosion of user generated content have resulted in an overwhelming amount of video material available online. However, to date, access to this wealth of information is hampered by the lack of good indexing mechanisms. At best, archivists provide a few, often subjective, keywords or tags - by far insufficient for a directed large-scale search. Likewise, jumping to the fragment of interest within a video is normally not possible, as the manually added tags are not time-coded, forcing users to fall back to random sampling of the video timeline or to feed forward search through the video in a linear fashion.

Nevertheless, video has the potential to become the medium of the future, being low-threshold, often languageindependent, direct and closer to 'real-life' than pure text - if only it could be accessed and retrieved as easily as text. All that is required to make this happen is detailed, time-coded annotations of the video content. So the question is: "How can we easily obtain good annotations ?”. In this work, we focus on the problem of naming the persons appearing in news videos.

Phi The Pham is a Ph.D. student at the Department of Computer Science at the Katholieke Universiteit Leuven, Heverlee B-3001 Belgium, e-mail: (see http://www.cs.kuleuven.be/ phi).

Tinne Tuytelaars is a research professor (BOF-ZAP) at the Department of Electrical Engineering at the Katholieke Universiteit Leuven, Heverlee B-3001 Belgium, e-mail: (see http://homes.esat.kuleuven.be/ tuytelaa).

Marie-Francine Moens is a research professor (BOF-ZAP) at the Department of Computer Science at the Katholieke Universiteit Leuven, Heverlee B-3001 Belgium, e-mail: (see http://www.cs.kuleuven.be/ sien).
In spite of the increased availability of crowdsourcing (see e.g. Amazon Mechanical Turk (http://www.mturk.com)), the scale of the problem is such that manual annotation is not an option. So instead, (semi-) automatic video analysis tools are being developed, with increasing success - see e.g. large scale initiatives such as Quaero (http://www.quaero.org) and Theseus (http://theseus-programm.de) in Europe, Informedia (http://www.informedia.cs.cmu.edu/) in the US, or the STAR challenge (http://www.thestarchallenge.sg/) organized in Singapore. In this endeavour, special care has to be taken to ensure the tools are scalable and easily kept up-to-date, in order to match the wide variety and variability in video content. This in turn calls for weakly supervised or semi-supervised methods, that can learn from noisy or partially labeled data.

Methods that learn from multimodal data (sometimes referred to as weak supervision in the context of image or video annotation) are becoming increasingly popular. By exploiting information from multiple modalities, the effort of constructing a large, carefully collected and annotated training corpus can often be avoided or reduced. Instead, it may suffice to refer to a trusted, relevant source of information, such as wikipedia pages, a news website containing images with captions, or other types of multimodal information to be used as training data with limited supervision. In this paper, we use available video transcripts, which actually correspond to time-coded subtitles. Similarly, semi-supervised methods are gaining in importance. In this case, only a small part of the training material is annotated. The remainder of the data set is not just ignored, but rather exploited in a clever way, in spite of the lack of labels. It has been shown that in classification tasks learning from labeled and unlabeled examples has a benefit on classification performance, especially when few labeled training examples are available [1], [2]. Here, we manually link each of the names appearing in the transcript to a couple of faces seen in the video.

People play an important role in most video content, and tools for identifying the individuals appearing in a video would be especially helpful. Appearance of people in a corpus typically follows a power law, with some people appearing very frequently, while the majority are very rare or do not appear in the corpus at all. At the same time, the appearance of a person's face can vary dramatically, due to changes in pose, changes in lighting conditions, facial expressions and partial occlusions.

Here, we label video with textual information extracted from the transcripts. This is a difficult problem, as there are often many faces in a frame, and many names in the transcripts of the video, as well as many unnamed faces or names that are 
mentioned but not displayed. In addition, subtitles usually do not directly describe the video content, but on the contrary are often complementary to the visual information. Moreover, even if the name of a face is explicitly mentioned, the temporal alignment may be relatively weak. As a result, learning to name faces from video subtitles is a more challenging problem than learning from image captions (as in [3], [4]). To make the quality of the results acceptable, we propose a semisupervised scheme, where a few faces of each person in the database are labeled manually, and this information is then propagated to similar unlabeled faces (see Figure 1). This is achieved using a graph based algorithm that learns the nameface alignments jointly from labeled and unlabeled examples. Moreover, by first identifying the news anchor(s), the overall performance can be improved significantly. This paper expands the preliminary results described in [5]. Variant versions of the label propagation model are proposed here that deal with time and space complexity of processing large datasets. In addition, we show the portability of labeled seeds across different broadcasts. Compared to [5] we evaluate and compare with state-of-the-art labeling approaches on a larger dataset, i.e., on 9 broadcasts of BBC news.

The remainder of this paper is organized as follows. We start with a description of related work. In section III we discuss the video data preprocessing, i.e., face detection and tracking in the video frames and detection and clustering of person names in the transcripts. The anchor detection in news broadcasts is the topic of section IV. Section V describes the label propagation algorithm that computes the probability of a name for each face. Section VI discusses the face similarity metric used. In section VII the experiments and results are given. Section VIII concludes the paper.

\section{RELATED WORK}

Several researchers have looked into the problem of linking names and faces in video, be it with a manual initialization (e.g. [6], [7], [8]) or fully automatic based on temporal cooccurrence of names and faces (e.g. [9], [10], [3]). The initial information of names and faces correspondence is then used as a training set for a regular face classification process [6], [10], a multiple instance learning process [7], or in a nearestneighbors framework [8].

Based on a large news photo with caption collection, [11] proposes a graph based method. They first use the queried name to collect a set of associated faces. Then a weighted graph is built where the nodes are the associated faces and the edges are their similarities. Finally, they find the densest component - a set of highly connected nodes of the graph, and consider this set of nodes to correspond to the faces of the queried person. To annotate a photo collection of different users taken over a period of 8 months, [12] first obtains a set of high confidence annotation labels using a supervised classifier. Then they propagate these labels to the remaining photos by a probabilistic label propagation method, which uses timing, location and visual proximity information. [13] solves a different problem (unsupervised object discovery), yet uses somewhat similar techniques: a graph is constructed, with all images as vertices and edge weights based on image similarity (number of feature matches). Then they use normalized cuts to partition the graph in different clusters ("objects"). All these methods use faces (or objects) as vertices of the graph. Here, we extend label propagation to video annotation. We also experiment with a graph over name-face pairs, which allows more complex constraints to be integrated easily.

The problem of anchor person detection is well studied, e.g. [14]. The common assumption is that anchor persons appear many times throughout the news broadcast and in front of the same studio settings. Hence, images are clustered based on their color similarity. Clusters with more than one member and with a total time span higher than a threshold are considered referring to anchor persons. Other information such as audio and face appearance have also been used to boost the anchor detection accuracy. In this paper, we do not only detect anchor persons, but also identify them with their name.

\section{VIDEO DATA PREPROCESSING}

Our methods presume video frames and related text transcripts. These are then processed with the goal of detecting faces, tracking the faces in subsequent frames, detecting person names in the transcripts and tracking the references to a person in the texts.

First, faces are detected using the OpenCV implementation of [15]. Next, we detect facial features [10] and use these as initial pose estimation for a 3D morphable face model [16] that is fitted to the data. Using such a 3D morphable model allows to estimate the pose and illumination parameters and to eliminate these irrelevant sources of variability. Also partial occlusions can be overcome this way. The model returns 40 person-specific texture components and 40 person-specific shape components, which together form the face descriptors $f_{i}^{k}$ used in this work.

A typical news footage lasts on average 30 minutes and contains around 30.000 detected faces. These faces arise from just a few hundred "tracks" of a particular character each in a single shot [10]. Applying a face-tracking method reduces the amount of data to be processed and allows to select the best faces in each face track. We use a KLT tracker [17] to track faces using similar point tracks over frames.

In the transcript, a first step is to recognize person names. We use a named entity recognizer which is based on a maximum entropy classifier. The classifier takes into account capitalization patterns of a term to decide whether the term represents a proper name and the context words to decide on the type of proper name. In this paper we are only interested in person names. The OpenNLP package (http://opennlp.sourceforge.net/) is used for this task, which we augment with a gazetteer of names, extracted from the Wikipedia website (http://en.wikipedia.org/). In a transcript several mentions (e.g., "Al Gore", "former vice president", "he") might refer to the same person and form a coreference chain. This noun phrase coreference resolution follows the methods of the LingPipe package (http://www.aliasi.com/lingpipe/). To group mentions of the same person across 


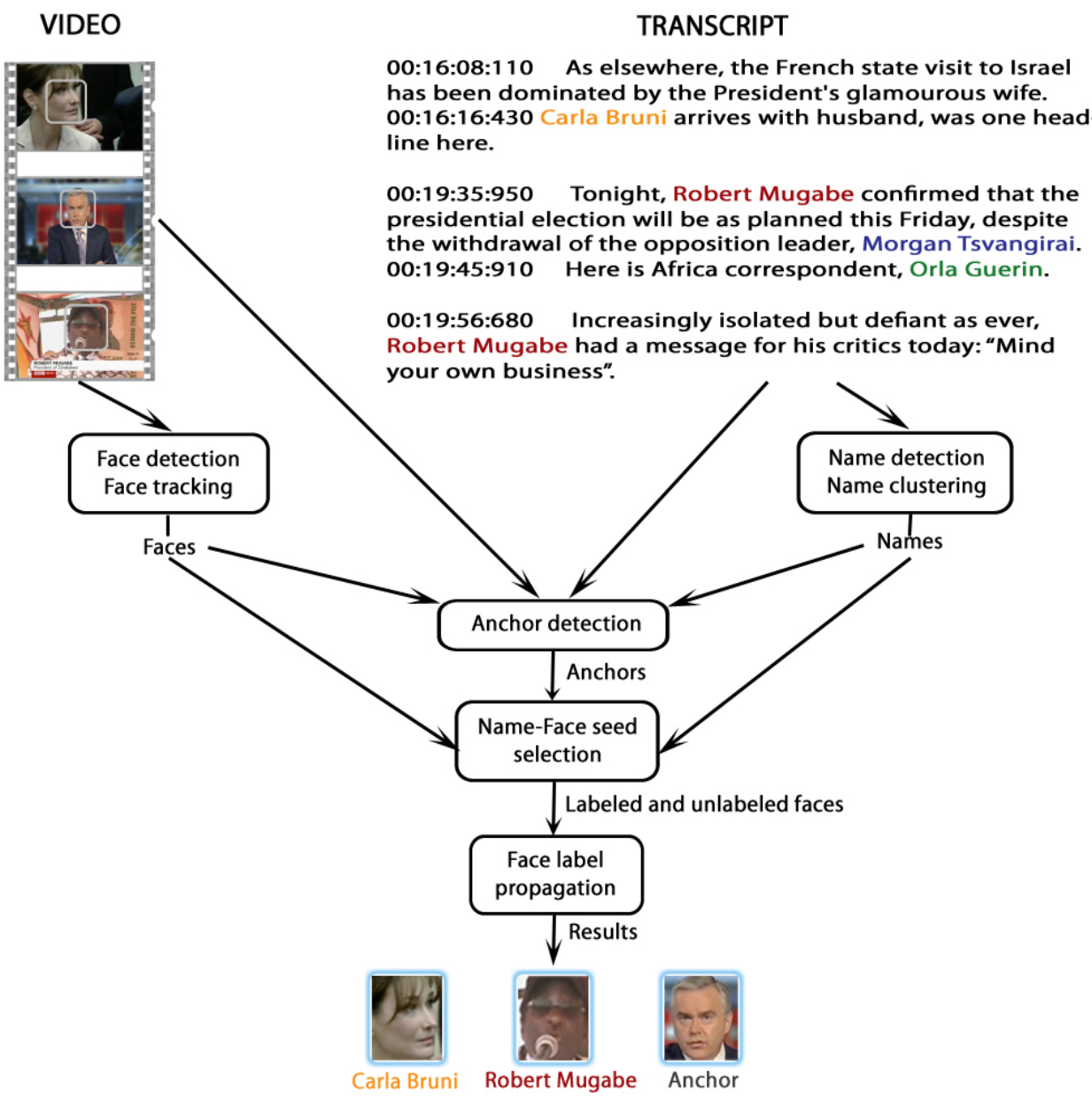

Fig. 1. The face label propagation process (source: BBC News).

the transcript, we use a dictionary of variant names built with a string edit distance function in combination with a clustering of the coreference chains of a name, where the latter allows to resolve mentions of the single word "Bush" to "George W. Bush" and not to "Laura Bush". Then coreference chains of each text are clustered with a hierarchical single link algorithm constrained by a threshold cosine similarity for cluster membership.

\section{ANCHOR DETECTION}

Anchors may be problematic, since their names are typically mentioned only once, at the very beginning of the news broadcast. Yet, anchors occur quite frequently. Rather than looking at the names and faces information only, we exploit the fact that anchors typically occur over a wide time range and against a typical background (e.g. the news studio). Figure 2 shows some typical examples.

We adapt D'Anna et al.'s approach to find the anchor face tracks based on scene similarity [14]. To find the names of the anchors, we use the transcript clues proposed in [6]. Finally, to match an anchor face track and a candidate name, we adapt the timing similarity approach by Satoh et al. [9]. Each of these steps is described next.
To find the anchor face tracks, we first find the key frames that (might) contain the anchors, and then decide which face tracks within these key frames refer to the anchors. We start by selecting, for each face track, the frame with the highest quality of face fitting as representative key frame. Next, we cluster the key frames, using a graph-theoretical clustering analysis. We build a graph $G$ with key frames as nodes and the weights of the edges set to the visual distances between the nodes (see below on how we compute these). Then, a minimum spanning tree $(M S T)$ is built on the graph $G$ using Kruskal's Algorithm [18]. In the $M S T$ all the edges with weight larger than a threshold are removed, resulting in a forest containing a number of smaller trees. From these, trees (or clusters) with small lifetime (i.e. the time interval that includes all the frames of the cluster) are discarded. Each of the remaining trees is considered as a cluster containing the frames of an anchor person.

Key frames are described by local color histograms computed over 16 rectangular non-overlapping regions covering the image. To compare key frames, the eight regions with the most similar histograms are selected and the distances between their color histograms summed. This procedure makes the frame similarity measure robust to variations such as the large screens 


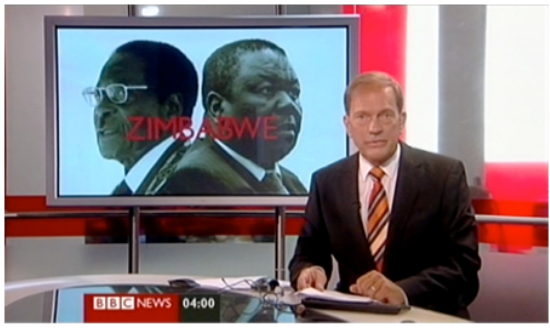

00:00:46:560 Hello, welcome to BBC, I'm Alastair Yates.

00:00:50:240 President George W. Bush has described Zimbabwe's election as a sham and he has called for strong action from the United Nations.

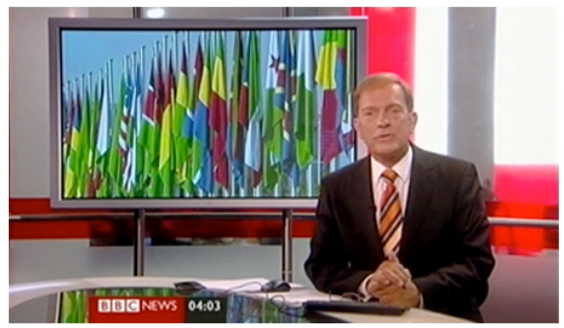

00:03:44:210 There have been calls for the AU to exclude Robert Mugabe on the grounds that he wasn't properly elected or to impose sanctions on Zimbabwe.

00:03:53:040 But it is hardly the style of the African Union, which prefers to smooth over conflict and look for consensus.

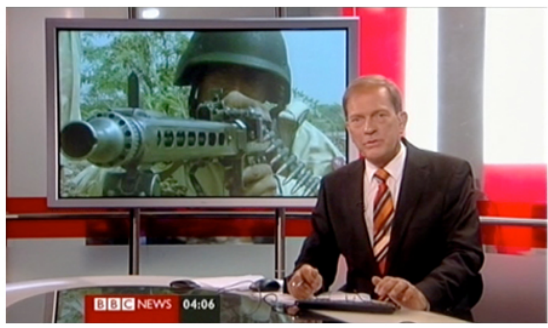

00:06:12:120 The Pakistani military has launched an operation against militants in the northern town of Pashar. 00:06:20:600 The group have been seeking to impose more Austere codes of Islamic conduct.

Fig. 2. Some example news video clips with the anchor (source: BBC News).

in figure 2. Histograms are compared using the distance measure similar to the one proposed by Giannakopoulos (http://www.mathworks.com/matlabcentral/fileexchange/22030image-retrieval-query-by-example-demo).

Not all faces appearing in the selected anchor frames actually belong to the anchor(s). Non-anchors are filtered out by clustering all the faces appearing in the anchor frames and removing clusters containing only one face.

For each anchor face track, the corresponding name is found using alignment according to the temporal appearance of a face track and a name. Let $\left(t_{f t}^{\text {start }}, t_{f t}^{\text {end }}\right)$ the start and end time of a face track $f t$. Let $t_{n}$ the time that name $n$ is mentioned. The temporal co-occurrence similarity of the face track $f t$ and the name $n$ can be defined as follows:

$$
\begin{gathered}
T S(f t, n)= \begin{cases}1 & \text { if } t_{f t}^{\text {start }} \leq t_{n} \leq t_{f t}^{\text {end }}, \\
\alpha & \text { otherwise. }\end{cases} \\
\alpha=\exp \left(-\frac{\left(\left(t_{f t}^{\text {start }}+t_{f t}^{\text {end }}\right) / 2-t_{\text {off }}-t_{n}\right)^{2}}{\left(\left(t_{f t}^{\text {end }}-t_{f t}^{\text {start }}\right) / 2\right)^{2}}\right)
\end{gathered}
$$

where $t_{o f f}$ is the average time lag between the time a name is mentioned untill its corresponding face appears.

Given an anchor face track - name pair $\langle f t, n\rangle$, if $T S(f t, n)$ is larger than a threshold, and the name $n$ is introduced in the form such as "I'm Alastair Yates" or "from $m e$, Alastair Yates" , the pair $\langle f t, n\rangle$ is selected. This pairing approach is similar to the "exemplars" concept used in [10]. All thresholds are set on a small held-out validation set.

In this framework, the named anchors are moved from the unlabeled face set to the training set. This will reduce the ambiguity in our name label propagation process and might improve the alignment performance.

\section{NAMING FACES WITH LABEL PROPAGATION}

\section{A. Presentation of the problem}

We first annotate a small number of name-face seed pairs. This annotation can be done manually by randomly selecting a number of faces for each name mentioned. Given this training set of labeled faces, the problem is choosing names for the unlabeled faces. In this paper, our interest goes to algorithms that learn jointly from the labeled and unlabeled faces, exploiting both visual and textual information. Among various classification methods using labeled and unlabeled data, label propagation by a random walk process approach is promising. We adapt the approach of [2] to formulate a framework of name label propagation over faces.

Suppose we initially have name labels for $l$ faces and the remaining $u$ faces do not have name labels yet. We denote $\left(f_{1}, n_{1}\right) \ldots\left(f_{l}, n_{l}\right)$ the set of labeled faces where $N_{l}=$ $\left\{n_{1} \ldots n_{l}\right\}, n_{i} \in\{1 \ldots C\}$ are the name labels. The constraints are: the number of distinct names $C$ is known and all the distinct name labels appear in the set of labeled faces. The set of unlabeled faces can be presented as $\left(f_{l+1}, n_{l+1}\right) \ldots\left(f_{l+u}, n_{l+u}\right)$ where the name labels $N_{u}=\left\{n_{l+1} \ldots n_{l+u}\right\}$ are not known yet. Let $F$ be the set of all faces $F=\left\{f_{1} \ldots f_{l+u}\right\} \in R^{D}$ described with $D$ features. We will use $F$ and $N_{l}$ to predict $N_{u}$.

If a group of faces is similar, they may have the same name. The level of similarity between these faces determines the confidence that they share the same name. Labeled faces contribute to the naming of an unlabeled face by their names with a confidence estimated by their similarities with this unlabeled face. Moreover, unlabeled faces also affect the labels of each other by their similarity or co-occurrence.

\section{B. Using solely face similarities}

To implement the above observation, we build a fully connected graph $G^{f}$ where the nodes are all $l+u$ faces. The weight $w_{i j}^{f}$ of the edge between faces $f_{i}$ and $f_{j}$ is the similarity between them, and the one-step transition probability $T_{i j}^{f}$ from face $j$ to face $i$ can be estimated from the edge weights:

$$
T_{i j}^{f}=P(j \rightarrow i)=\frac{w_{i j}^{f}}{\sum_{k=1}^{l+u} w_{k j}^{f}}
$$

All faces have probability distributions over name labels. We define a probability matrix $N^{f}$ of size $(l+u) \times C$ where row $N_{i}^{f}$ represents the probability distribution over all name labels for face $i$. 


\section{Using name-face pairs similarities}

In videos, we notice that faces in the same frame almost always have different names; while faces in the same face track must have the same name. These extra constraints can be exploited, by building a graph $G^{n f}$ of name-face pairs. To this end, we define the following additional functions (corresponding to "same name", "same frame", and "same appearance" respectively):

$$
\begin{gathered}
S N\left(n_{x}, n_{y}\right)= \begin{cases}1 & \text { if } n_{x}=n_{y}, \\
0 & \text { otherwise. }\end{cases} \\
S F\left(f_{a}, f_{b}\right)= \begin{cases}1 & \text { if } f_{a} \text { and } f_{b} \text { are } \\
\text { in the same video frame, } & \text { otherwise. }\end{cases} \\
S A\left(f_{a}, f_{b}\right)= \begin{cases}1 & \text { if } f_{a} \text { and } f_{b} \\
\text { are in the same face track, } & \text { thus have the same appearance, } \\
\operatorname{sim}_{f_{a}, f_{b}} & \text { otherwise. }\end{cases}
\end{gathered}
$$

In equation $6, S A\left(f_{a}, f_{b}\right)$ makes use of the results of the face tracking process in the sense that if two faces are in the same face track, their similarity is set to 1 reflecting that the probability they share the same name is very high.

In this model we consider all candidate name-face alignment pairs of one news broadcast, some of which are sure alignments (labeled examples) while for the other pairs we do not know if the alignment holds. We build a new graph $G^{n f}$, now with the name-face pairs as vertices and a new transition matrix $T_{i j}^{n f}$ between name-face pairs. From the above constraints, we define the weight $w_{i j}^{n f}$ of the edge connecting the name-face pairs $i=<f_{a}, n_{x}>$ and $j=<f_{b}, n_{y}>$ as follows:

$$
w_{i j}^{n f}=S N\left(n_{x}, n_{y}\right) *\left(1-S F\left(f_{a}, f_{b}\right)\right) * S A\left(f_{a}, f_{b}\right)
$$

The one-step transition probability $T_{i j}^{n f}$ from name-face pair $j$ to name-face pair $i$ can again be estimated from the edge weights:

$$
T_{i j}^{n f}=P(j \rightarrow i)=\frac{w_{i j}^{n f}}{\sum_{k=1}^{(l+u) C} w_{k j}^{n f}}
$$

All name-face pairs now have a probability in the probability matrix $N^{n f}$ indicating the strength of their association.

\section{The label propagation algorithm}

After setting up the graph $G^{*}$ (either $G^{f}$ or $G^{n f}$ ), the transition matrix $T^{*}$ and the label matrix $N^{*}$, we perform the label propagation algorithm as follows:

1) All faces/name-face pairs propagate labels for one step: $N^{*} \leftarrow T^{*} N^{*}$

2) Row normalize $N^{*}$ to maintain the label probability interpretation.

3) Clamp the labeled faces. Repeat from step 1 until convergence of $N^{*}$.
Step 3 is important since it ensures the names of the labeled faces are kept. Via iterations, the labels are propagated through the high density (similar) face/name-face pair regions (clusters) while the propagation slows down in the low density gaps between face/name-face pair clusters. The label propagation algorithm is proved to converge [2].

\section{E. Face naming after the label propagation process}

Matrix $N^{*}$ then contains the label distribution for each face/name-face pair. In the experiments below we choose for each unlabeled face the name with highest probability given the face, or in case of name-face pairs, the name of the nameface pair that has highest probability given the face. We select names when the probability is above a threshold $\lambda$ (called name assignment threshold in the experiments below) [10]. This refusal to predict strategy leaves some faces unlabeled - in other words these faces refer to the null name. In case of the label propagation using the graph of name-face pairs, the seed name-face pair with which a name-face pair has the highest probability in $N^{*}$ is chosen for labeling the unknown face.

\section{F. Reducing the computational complexity}

The space complexity of $T^{*}$ is a quadratic function of the number of faces. To deal with this problem, we have experimented with an approach that only uses the face track information, and an approach that implements a sparse matrix. In the first approach, each node in the graph, instead of being a face/name-face pair, is represented by a face track/name-face track pair. We define the edge weight $w_{i j}^{t}$ between two face tracks $t_{i}$ and $t_{j}$ as the maximum similarity between the faces in $t_{i}$ and in $t_{j}$ :

$$
w_{i j}^{t}=\max _{f_{p} \in t_{i}} \max _{f_{q} \in t_{j}} \operatorname{sim}_{f_{p}, f_{q}}
$$

The weight $w_{i j}^{n t}$ of the edge connecting the name-face track pairs $i=<t_{a}, n_{x}>$ and $j=<t_{b}, n_{y}>$ is defined as follows:

$$
w_{i j}^{n t}=S N\left(n_{x}, n_{y}\right) * S A\left(t_{a}, t_{b}\right)
$$

with $S N\left(n_{x}, n_{y}\right)$ defined as before and

$$
S A\left(t_{a}, t_{b}\right)= \begin{cases}0 & \text { if } t_{a} \text { and } t_{b} \text { contain at least } \\ & \text { two faces in the same video frame } \\ w_{a b}^{t} & \text { otherwise }\end{cases}
$$

By considering the tracks, the number of nodes in the graph is significantly reduced.

In the second approach we restrict a face to be linked with at most $k_{f}$ nearest neighbor faces and a face track to be linked with at most $k_{t}$ nearest neighbor face tracks. This makes $T^{*}$ a real sparse matrix, which we can store efficiently. 


\section{LEARNING A PARAMETER FOR THE FACE SIMILARITY FUNCTION}

The last problem is to learn a good measure for face similarity. A simple function to estimate the similarity between two faces $f_{i}$ and $f_{j}$ is defined as follows:

$$
\operatorname{sim}_{f_{i} f_{j}}=\exp \left(-d_{f_{i} f_{j}}\right)=\exp \left(-\sqrt{\left.\sum_{k=1}^{D}\left(f_{i}^{k}-f_{j}^{k}\right)^{2}\right)}\right.
$$

This exponential similarity function is chosen for its simplicity and suitability for image and feature spaces (here, human faces). Moreover, our face descriptors $f_{i}^{k}$ are obtained with principal component analysis, so they are uncorrelated. The function has a fast decreasing rate with increasing face distances. Hence, only close-by faces are connected and propagation is limited to really similar faces. This partially satisfies our desire. Because of their variations in expressions, occlusions, and unmodeled lighting changes (e.g. specularities), not only close-by faces refer to the same person. To moderate the decreasing rate and balance the nearby face connections against the far away face connections, we incorporate a parameter $\sigma$ into this similarity function:

$$
\operatorname{sim}_{f_{i} f_{j}}=\exp \left(-\frac{d_{f_{i} f_{j}}^{2}}{\sigma^{2}}\right)=\exp \left(-\frac{\sum_{k=1}^{D}\left(f_{i}^{k}-f_{j}^{k}\right)^{2}}{\sigma^{2}}\right)
$$

$\sigma$ needs to be optimized so that related faces receive higher similarity values than dissimilar faces. For each broadcast, we find $\sigma$ using its labeled faces $N_{l}$. First, we construct a minimum spanning tree $M S T$ over all labeled faces given the Euclidean distances $d_{f_{i} f_{j}}$ between faces. Then, we search through the $M S T$ for the edge with shortest distance that connects two faces with different labels and get the length $d^{0}$ of this edge. We assume $d^{0}$ as the minimum distance between face clusters. Following the $3 \sigma$ rule of the Normal distribution, we set $\sigma=\frac{d^{0}}{3}$. By using the newly found $\sigma$ value in estimating the faces similarity, we hope that the similarity of faces within one name class is high and of faces between different name classes is low. This enforces strong label sharing between faces within one cluster and weak label sharing between faces from different clusters. The $M S T$ is again constructed with Kruskal's Algorithm.

We have also experimented with metric learning for estimating the face similarities following [4], but this did not improve the final face labeling results.

\section{EXPERIMENTS}

\section{A. Evaluation specifics}

The performance of the face labeling process is expressed in terms of Precision versus Recall. Precision is the proportion of the correctly recognized faces in all recognized faces, while Recall is the proportion of faces which are assigned a name after the "refusal to predict" mechanism (see section V-E). We adopt Everingham et al.'s approach [10] to evaluate our face labeling method, varying the name assignment threshold.

\section{B. Datasets and preprocessing}

We perform and compare our experiments on nine BBC afternoon news broadcasts recorded from 22-Jun-2008 to 01Jul-2008. Each broadcast lasts approximately 30 minutes, or 60.000 frames.

After the face detection and tracking process, we obtain from each broadcast an average of 28.000 faces, forming on average 120 face tracks. To reduce the number of false positives in the face detection, we filter out the detections with a too low confidence value.

Finally, to further reduce the processing time, an average of 3 representative faces are selected for each track. These kept faces are first selected by their size (the larger, the better), then by their fitting confidence. After reduction, a broadcast contains on average 496 faces and 116 face tracks.

For the name detection and clustering, we obtain from each broadcast an average of 21 unique candidate names. On average, $81 \%$ of the face tracks detected in the broadcasts are not the anchors.

The detailed data preprocessing statistics are shown in table I(a).

\section{Anchor detection}

Table I(b) shows the anchor face track detection performance. The overall anchor detection precision is high $(88 \%$ on average).

This is helpful to the later label propagation process, where the quality (i.e., precision) of the training examples is more important than the quantity (i.e., recall).

\section{Forming the training set}

After the face filtering and reduction process, from the set of candidate names, each distinct candidate name is used to label up to 6 faces if available (with random face selection). Note that many names are mentioned in the transcript but do not have corresponding faces in the video frames, so they are assigned to the null face. Table I(c) shows the number of manually labeled faces per news broadcast. The number of labeled faces varies among the broadcasts, since the number of names is different per broadcast.

\section{E. Label propagation}

We use the label propagation algorithm in two settings: 1) when the nodes of the graph represent the faces, and the edges are weighted with the face similarities $(F)$, and 2 ) where the nodes of the graph represent name-face pairs, and the edges are weighted with similarities between nameface pairs $(N F)$. The latter case allows integrating name-face constraints. We evaluate the label propagation in three settings: (1) without parameter learning (i.e., using eq. 12) and without anchor detection (denoted as $N P-N A$ ); (2) with parameter learning but without anchor detection (WP-NA); and (3) with both parameter learning and anchor detection (WP-WA). The label propagation process is applied on each news broadcast separately. Then, the final face labeling performance is the 
(a) Data preprocessing statistics. NDF: Number of detected faces; NDFT: number of detected face tracks; NRF: number of faces after data reduction; NRFT: number of face tracks after data reduction; NDN: number of distinct detected names.

\begin{tabular}{|l||l||l||l||l||l|}
\hline Broadcast & NDF & NDFT & NRF & NRFT & NDN \\
\hline BBC 22-Jun-2008 & 31275 & 129 & 435 & 125 & 17 \\
\hline BBC 24-Jun-2008 & 22766 & 131 & 402 & 118 & 26 \\
\hline BBC 25-Jun-2008 & 11420 & 53 & 177 & 49 & 13 \\
\hline BBC 26-Jun-2008 & 31279 & 122 & 398 & 119 & 25 \\
\hline BBC 27-Jun-2008 & 38487 & 169 & 670 & 168 & 32 \\
\hline BBC 28-Jun-2008 & 31503 & 120 & 334 & 115 & 32 \\
\hline BBC 29-Jun-2008 & 33163 & 139 & 451 & 137 & 10 \\
\hline BBC 30-Jun-2008 & 25095 & 100 & 308 & 97 & 17 \\
\hline BBC 01-Jul-2008 & 27579 & 123 & 390 & 119 & 22 \\
\hline
\end{tabular}

(b) Anchor face track detection performance. NDA: Number of detected anchor face tracks; NGT: Number of ground truth anchor face tracks; NCR: Number of correctly detected anchor face tracks; P: Precision; R: Recall.

\begin{tabular}{|l||l||l||l||l||l||l|}
\hline Broadcast & NDA & NGT & NCR & P (\%) & R (\%) & F1 (\%) \\
\hline BBC 22-Jun-2008 & 30 & 36 & 30 & 100 & 83.33 & 90.91 \\
\hline BBC 24-Jun-2008 & 18 & 18 & 14 & 77.78 & 77.78 & 77.78 \\
\hline BBC 25-Jun-2008 & 2 & 2 & 0 & 0 & 0 & N/A \\
\hline BBC 26-Jun-2008 & 22 & 25 & 18 & 81.82 & 72.00 & 76.60 \\
\hline BBC 27-Jun-2008 & 18 & 18 & 17 & 94.44 & 94.44 & 94.44 \\
\hline BBC 28-Jun-2008 & 8 & 20 & 8 & 100 & 40.00 & 57.14 \\
\hline BBC 29-Jun-2008 & 21 & 29 & 21 & 100 & 72.41 & 84.00 \\
\hline BBC 30-Jun-2008 & 29 & 29 & 22 & 75.86 & 75.86 & 75.86 \\
\hline BBC 01-Jul-2008 & 13 & 22 & 13 & 100 & 59.09 & 74.29 \\
\hline
\end{tabular}

(c) NLF: Number of manually labeled faces.

TABLE I

THE STATISTICS OF DATA PREPROCESSING, ANCHOR DETECTION AND FORMING OF THE TRAINING SET.

average of the respective recall and precision values (for the unlabeled faces) on all the broadcasts.

To clearly assess the label propagation, we compare the results with the results of a Support Vector Machine (SVM) (optimized with an RBF kernel) and a nearest neighbor classifier (NN) (similar to a k-means clustering but with the centroids fixed based on the labeled seeds). In this experiment we exclude anchor detection, and give results when using solely face similarities and when incorporating the same constraints as in the name-face pair label propagation.

Figure 3 and table II show that the label propagation algorithms in most cases outperform the SVM and NN classifiers.

The parameter learning for computing the face similarity does not seem to have a big impact. The anchor detection on the other hand seems very helpful in boosting the performance, especially when working with face tracks. Moreover the results show that label propagation using the name-face pair similarities yields better performance. For some news broadcasts and when using name-face pairs we observed that the incorporation of the named anchors did not outperform the other methods since it introduces many training examples of some anchor name classes creating a bias towards the anchor classes.

Figure 4 shows a representative set of clusters of faces grouped by their labeled names. From these examples, we see some important properties from both the data and the label propagation method: 1) Some faces appear frequently, especially the anchors (see Alastair Yates and Susan Osman), while others are rare since they are only shortly mentioned in the news. This problem of unbalanced face distribution clearly challenges the label propagation's final accuracy. As we can see from section V-E, after the matrix $N^{*}$ is computed, we take the most likely name for each unlabeled face as its name. However, the procedure does not provide any control over the final proportion of the name classes. As discussed in [2], we can incorporate the class proportions into the naming process, assuming that the class proportions can be estimated from the labeled data or known a priori (i.e. from an oracle). Both of these approaches require a lot more supervision and are opposed to our desire to use as little supervision as possible. 2) The lighting and poses of the faces vary significantly. This has consequences. First, the face detection and fitting process may be affected. Second, the accuracy of the face similarity estimation function drops. The combination of these two problems clearly affects the final performance of the label propagation process.

To reduce the temporal and spatial complexity we perform the same experiments, now solely relying on the face tracks. The results are shown in figure $3(\mathrm{c}, \mathrm{d})$ and table II(c,d), where $T$ refers to the face tracks and $N T$ to the name-face track pairs. The results are very good yielding up to more than $86.3 \%$ precision at $70 \%$ recall when using name-face track pair similarities, parameter learning and anchor detection, and in all settings the label propagation outperforms the SVM and NN classifiers. When combining the face track and the sparse matrix approaches, the results hardly decline and still show $85.8 \%$ precision at $70 \%$ recall using name-face track pair similarities, parameter learning and anchor detection. In this case, as discussed in section V-F, we set for each face the number of nearest neighbors $k_{f}=90$ and for each face track, the number of nearest neighbors $k_{t}=60$. In this setting memory usage and processing time are respectively almost 
(a) Label propagation with solely face similarities.

\begin{tabular}{|l||l||l||l||l|}
\hline Recall & $50 \%$ & $70 \%$ & $90 \%$ & $100 \%$ \\
\hline F-NP-NA & 70.36 & 64.52 & 53.16 & 49.25 \\
\hline F-WP-NA & 71.28 & 60.99 & 52.59 & 47.63 \\
\hline F-WP-WA & 75.84 & 66.23 & 61.87 & 54.60 \\
\hline F-SVM & 67.20 & 59.66 & 50.88 & 47.87 \\
\hline F-NN & 52.36 & 47.50 & 40.91 & 38.40 \\
\hline
\end{tabular}

(c) Label propagation with face track similarities.

\begin{tabular}{|l||l||l||l||l|}
\hline Recall & $50 \%$ & $70 \%$ & $90 \%$ & $100 \%$ \\
\hline T-NP-NA & 68.47 & 57.69 & 50.77 & 50.67 \\
\hline T-WP-NA & 67.57 & 51.95 & 52.24 & 49.78 \\
\hline T-WP-WA & NA & 82.76 & 80.00 & 73.20 \\
\hline T-SVM & 56.64 & 54.78 & 50.51 & 47.09 \\
\hline T-NN & 54.46 & 50.97 & 45.00 & 42.45 \\
\hline
\end{tabular}

QUANTITATIVE PRECISION RESULTS AT DIFFERENT RECALL LEVELS FOR THE NINE BBC NEWS BROADCASTS.

(b) Label propagation with name-face pair similarities.

\begin{tabular}{|l||l||l||l||l|}
\hline Recall & $50 \%$ & $70 \%$ & $90 \%$ & $100 \%$ \\
\hline NF-NP-NA & 80.53 & 70.54 & 56.69 & 52.67 \\
\hline NF-WP-NA & 80.59 & 67.40 & 58.95 & 52.52 \\
\hline NF-WP-WA & 81.92 & 72.66 & 66.80 & 60.14 \\
\hline NF-SVM & 76.60 & 67.79 & 58.26 & 53.82 \\
\hline NF-NN & 57.33 & 52.60 & 46.97 & 44.53 \\
\hline
\end{tabular}

(d) Label propagation with name-face track pair similarities.

\begin{tabular}{|l||l||l||l||l|}
\hline Recall & $50 \%$ & $70 \%$ & $90 \%$ & $100 \%$ \\
\hline NT-NP-NA & 76.15 & 55.77 & 50.75 & 49.78 \\
\hline NT-WP-NA & 68.70 & 55.48 & 53.00 & 49.78 \\
\hline NT-WP-WA & NA & 86.29 & 80.89 & 74.40 \\
\hline NT-SVM & 61.06 & 57.79 & 52.00 & 47.09 \\
\hline NT-NN & 56.25 & 51.28 & 45.54 & 41.70 \\
\hline
\end{tabular}

ABLE II

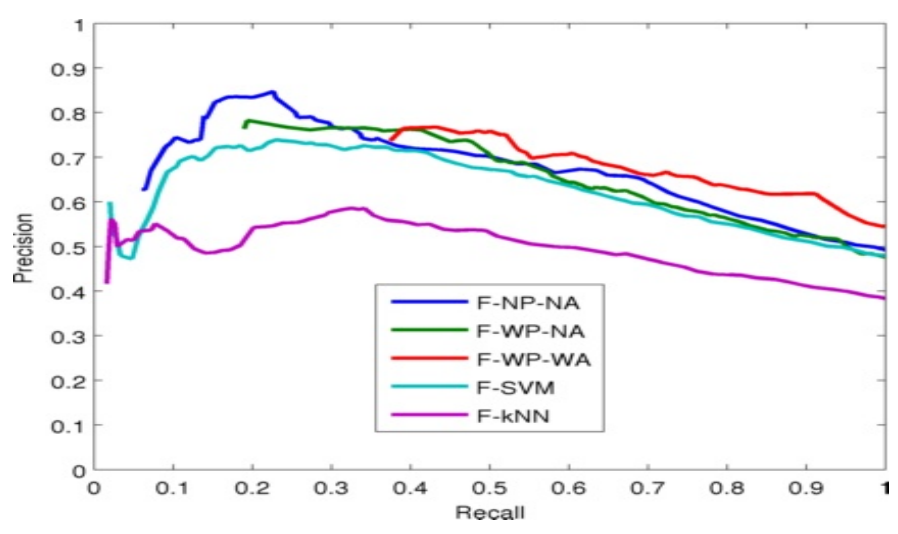

(a) Label propagation with solely face similarities.

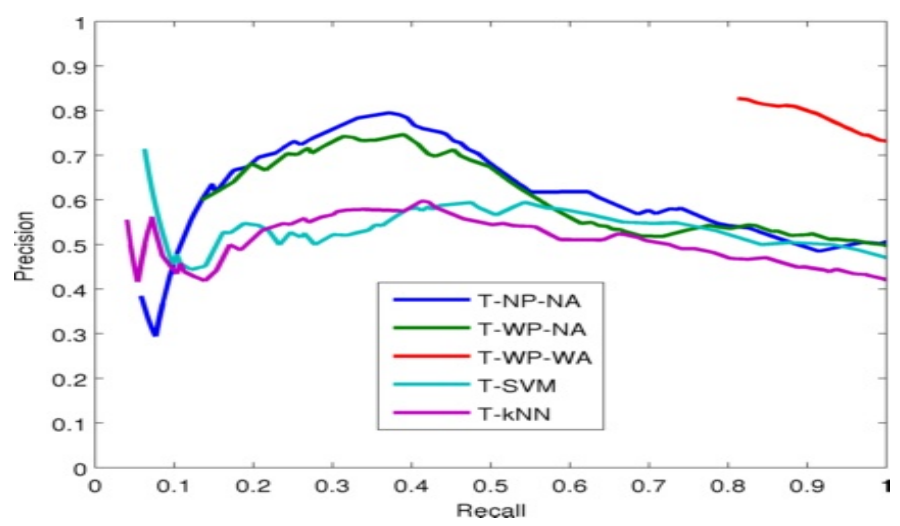

(c) Label propagation with face track similarities.

Fig. 3. Precision/recall curves averaged over the nine BBC news broadcasts.

15 times and about 7 times reduced.

In a final experiment, we test the portability of labeled faces across broadcasts with the proposed method. The label propagation framework perfectly allows the integration of labeled faces annotated for another broadcast in the transition probability matrix $T^{*}$. In this way labeled faces can be reused across broadcasts. On average per broadcast, $50 \%$ of the labeled faces are from persons also appearing in other broadcasts. Using anchor detection, name-face track pairs similarities and parameter learning, and importing training

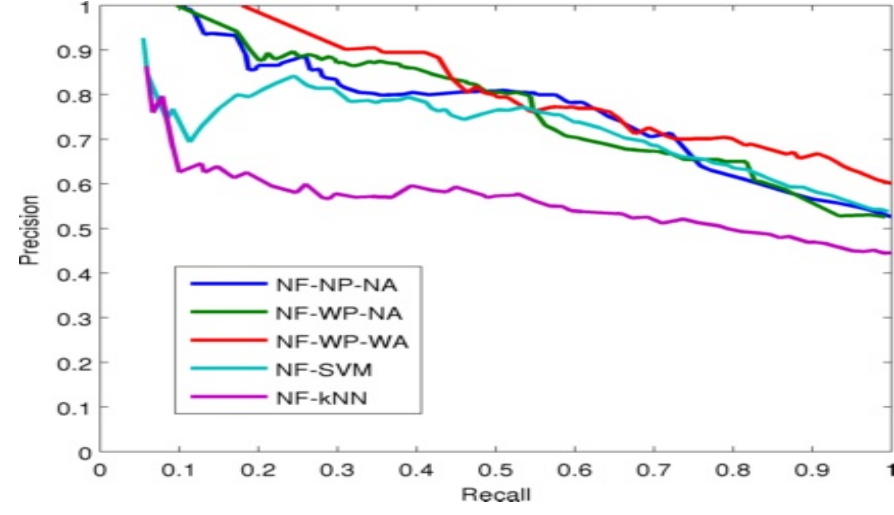

(b) Label propagation with name-face pair similarities.

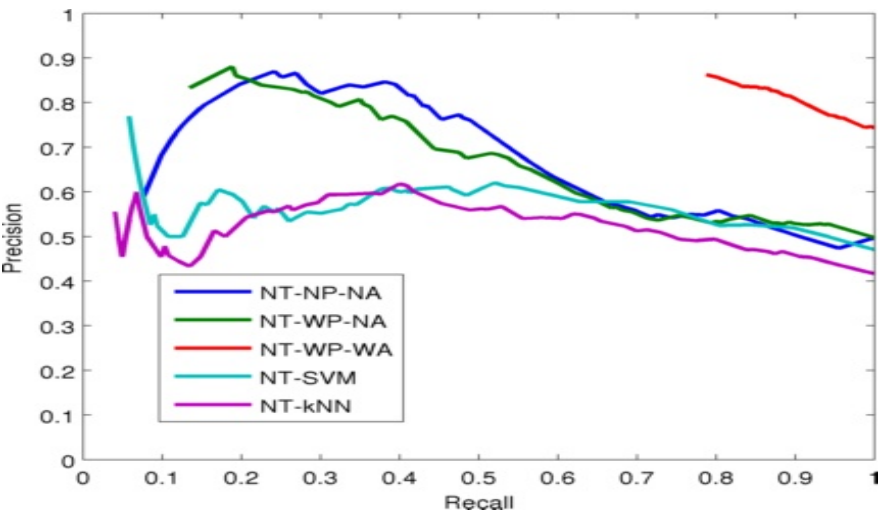

(d) Label propagation with name-face track pair similarities. data from the other broadcasts (with at most two labeled face tracks per name), we manage to name $70 \%$ of the faces with a precision of more than $85 \%$. The results show that, given a known set of labeled data, the label propagation mechanism can perform well on new unlabeled data. Importing labeled faces from other news broadcasts leads to a larger variation in face appearance. The face similarity parameter learning (see section VI) helps to cope with this. 

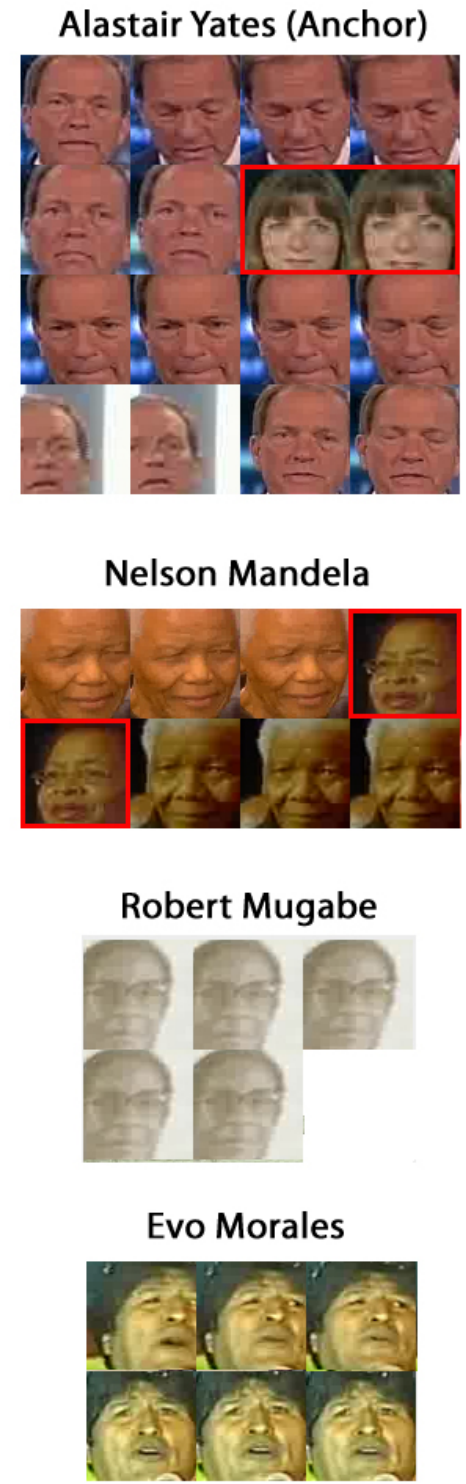
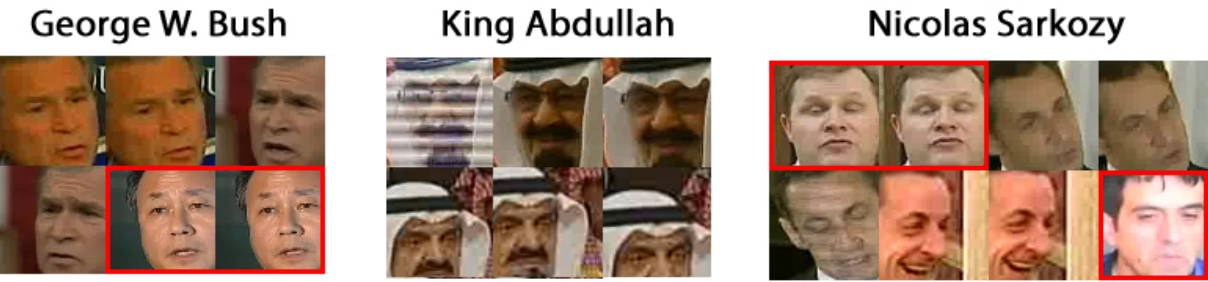

Hillary Clinton

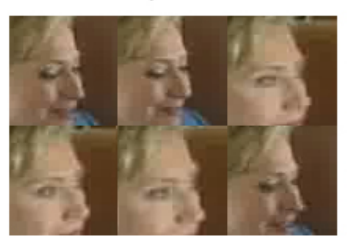

Barack Obama
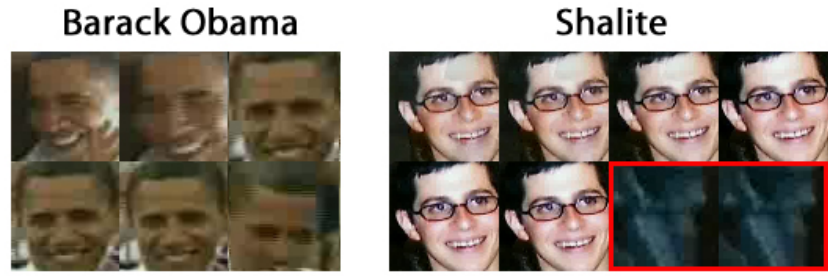

Gordon Brown
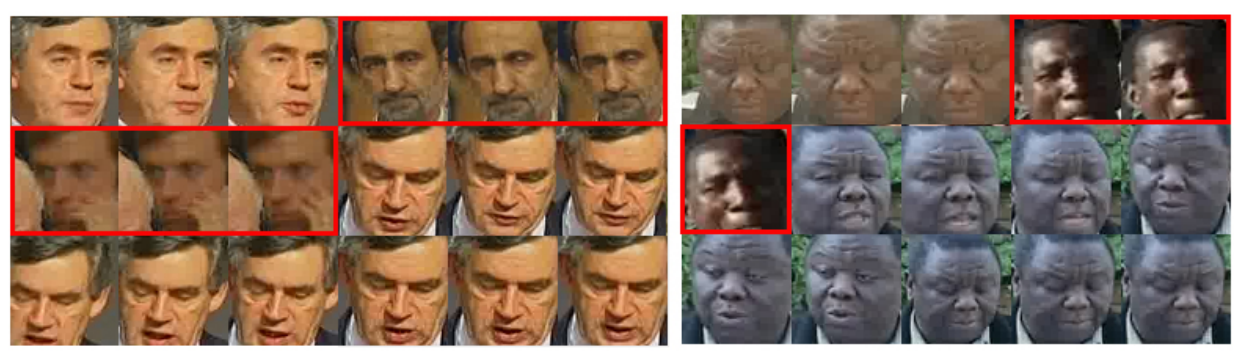

Susan Osman (Anchor)

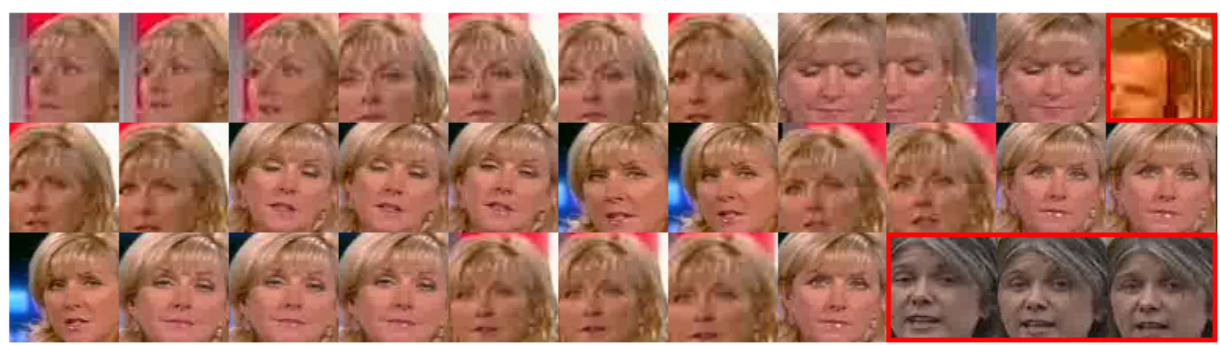

Fig. 4. A representative example of face labeling results with the faces wrongly labeled shown in the red box (source: BBC News).

\section{CONCLUSION}

We designed, implemented and evaluated a method for naming faces in video broadcasts that learns from labeled and unlabeled examples using iterative label propagation in a graph of connected faces or connected name-face pairs. When using a limited number of labeled faces, the label propagation algorithm outperforms a Support Vector Machine and a nearest neighbor classifier trained on the same labeled data. This performance is also apparent when replacing individual faces by their face track in order to reduce the computational complexity, and when reusing labeled faces from other broadcasts.

\section{ACKNOWLEDGMENT}

The research was financed by the IWT (SBO 060051) project AMASS++ (Advanced Multimedia Alignment and Structured Summarization). Tinne Tuytelaars was partially funded by the Fund for Scientific Research Flanders. The authors would like to thank Koen Deschacht and Wim De Smet for their valuable comments.

\section{REFERENCES}

[1] T. Zhang and F. J. Oles, "A probability analysis on the value of unlabeled data for classification problems," in Proc. 17th International Conf. on Machine Learning, 2000, pp. 1191-1198.

[2] X. Zhu and Z. Ghahramani, "Learning from labeled and unlabeled data with label propagation," Tech. Rep., 2002.

[3] T. Berg, A. Berg, J. Edwards, M. Maire, R. White, Y. Teh, E. LearnedMiller, and D. Forsyth, "Names and faces in the news," in Proceedings of the 2004 IEEE Conference on Computer Vision and Pattern Recognition, vol. 2, 2004, pp. 848-854.

[4] M. Guillaumin, J. Verbeek, and C. Schmid, "Is that you? Metric learning approaches for face identification," in International Conference on Computer Vision, sep 2009, pp. 498-505.

[5] P. T. Pham, M.-F. Moens, and T. Tuytelaars, "Naming persons in news video with label propagation," in Multimedia and Expo (ICME), 2010 IEEE International Conference on, 2010, pp. 1528 -1533.

[6] J. Yang and A. G. Hauptmann, "Naming every individual in news video monologues," in Proceedings of the ACM Multimedia 2004, October 2004, pp. 580-587. 
[7] J. Yang, R. Yan, and A. G. Hauptmann, "Multiple instance learning for labeling faces in broadcasting news video," in Multimedia '05: Proceedings of the 13th Annual ACM International Conference on Multimedia. ACM, 2005, pp. 31-40.

[8] D. Ramanan, S. Baker, and S. Kakade, "Leveraging archival video for building face datasets," in Proceedings of the 2007 IEEE International Conference on Computer Vision. IEEE Computer Society, 2007, pp. $1-8$.

[9] S. Satoh, Y. Nakamura, and T. Kanade, "Name-it: Naming and detecting faces in news videos," IEEE Mutimedia, vol. 1, pp. 22-35, 1999.

[10] M. Everingham, J. Sivic, and A. Zisserman, "Hello! My name is... Buffy - Automatic naming of characters in tv video," in Proceedings of the British Machine Vision Conference, 2006.

[11] D. Ozkan and P. Duygulu, "A graph based approach for naming faces in news photos," in CVPR '06: Proceedings of the 2006 IEEE Computer Society Conference on Computer Vision and Pattern Recognition. IEEE Computer Society, 2006, pp. 1477-1482.

[12] L. Cao, J. Luo, and T. S. Huang, "Annotating photo collections by label propagation according to multiple similarity cues," in Proceedings of the 16th ACM International Conference on Multimedia. New York, NY, USA: ACM, 2008, pp. 121-130.

[13] K. Grauman and T. Darrell, "Unsupervised learning of categories from sets of partially matching image features," in Proceedings of the IEEE Conference on Computer Vision and Pattern Recognition (CVPR), 2006.

[14] L. D'Anna, G. Marrazzo, G. Percannella, C. Sansone, and M. Vento, "A multi-stage approach for anchor shot detection," in Structural, Syntactic, and Statistical Pattern Recognition. Springer Berlin / Heidelberg, 2006, vol. 4109, pp. 773-782.

[15] P. Viola and M. Jones, "Robust realtime object detection vector quantization," International Journal of Computer Vision, vol. 57, no. 2, pp. 137-154, 2004.

[16] M. Desmet, R. Fransens, and L. Van Gool, "A generalized EM approach for 3D model based face recognition under occlusions," in Proceedings IEEE Conference on Computer Vision and Pattern Recognition, vol. 2, 2006, pp. 1423-1430.

[17] J. Shi and C. Tomasi, "Good features to track," in Proceedings of the Conference on Computer Vision and Pattern Recognition. IEEE Computer Society Press, June 1994, pp. 593-600

[18] J. B. Kruskal, "On the shortest spanning subtree of a graph and the traveling salesman problem," American Mathematical Society, vol. 7, no. 1 , pp. 48-50, 1956.

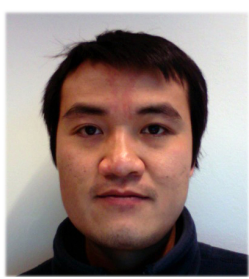

Phi The Pham is a research assistant at the Language Intelligence and Information Retrieval research group (HMDB-LIIR) of the Katholieke Universiteit Leuven, Belgium. He received a BS in 1998 from Can Tho University, Vietnam and ME in 2007 from the Katholieke Universiteit Leuven, Belgium. His research interests include multimedia and crosslingual information retrieval.

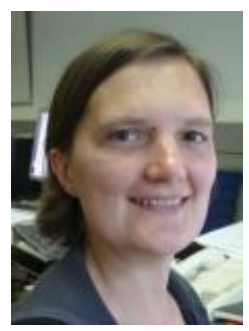

Tinne Tuytelaars is research professor (BOF-ZAP) at the Katholieke Universiteit Leuven, Belgium. She received a Master of Electrical Engineering from the K.U.Leuven in 1996. Since then, she has mostly been working at the VISICS-lab within ESAT - PSI of the K.U.Leuven, where she received her $\mathrm{PhD}$ on December 2000, entitled "Local Invariant Features for Registration and Recognition". Her main research interests include image representations, object recognition, and multi-modal analysis (combining images and text). She has published over 60 articles in peer-reviewed conferences and journals. She is member of the editorial board of Computer Vision and Image Understanding and Associate Editor of IEEE Transactions on Pattern Analysis and Machine Intelligence and serves as program committee member or area chair of the major computer vision conferences ICCV, ECCV, and CVPR.

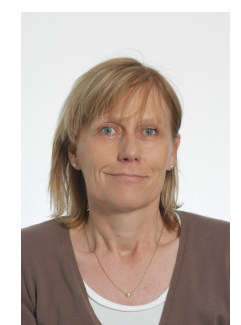

Marie-Francine Moens is a research professor (BOF-ZAP) at the Katholieke Universiteit Leuven, Belgium. She received a $\mathrm{PhD}$ in Computer Science in 1999 from this university. She leads a research team specialized in information retrieval and text analysis. She is author of two monographs published by Springer and numerous articles in proceedings of international conferences and journals. She is involved in the organization or program committee of major conferences on computational linguistics and information retrieval (ACL, SIGIR, EACL, ECIR, CIKM). She is the current chair-elect of the European Chapter of the Association for Computational Linguistics. 